\title{
Segmenting Liver Volume for Surgical Analysis
}

Ayman Al-Kababj

Faycal Bensaali

Sarada Prasad Dakua

\section{O):- Introduction}

(1) Background

- Two million people around the world die from hepatic-related diseases annually.

- In many cases, a liver hepatectomy is the solution to save a person life.

- To increase the chances of a successful hepatectomy, surgical pre-planning is necessary.

- 3D visualization can aid surgeons in understanding disease progression and for surgical planning.

\section{(C) Aims and Objectives}

- Create a 3D rendered liver volume, using machine learning, can help surgeons in visualizing the liver pre-operation.

- Delineate tumors within the 3D rendered volume.

- Delineate significant vessels in the 3D rendered volume.

- Create a script to segment liver tissues with a click of a button.

\section{Results}

- U-Net ConvNet is used for segmenting the liver, where a $2.5 \mathrm{D}$ input (5 slices) of pre-processed images are inserted into the ConvNet. Training environment is implemented on PyTorch using 5-fold cross validation with 80:20 train / val ratio

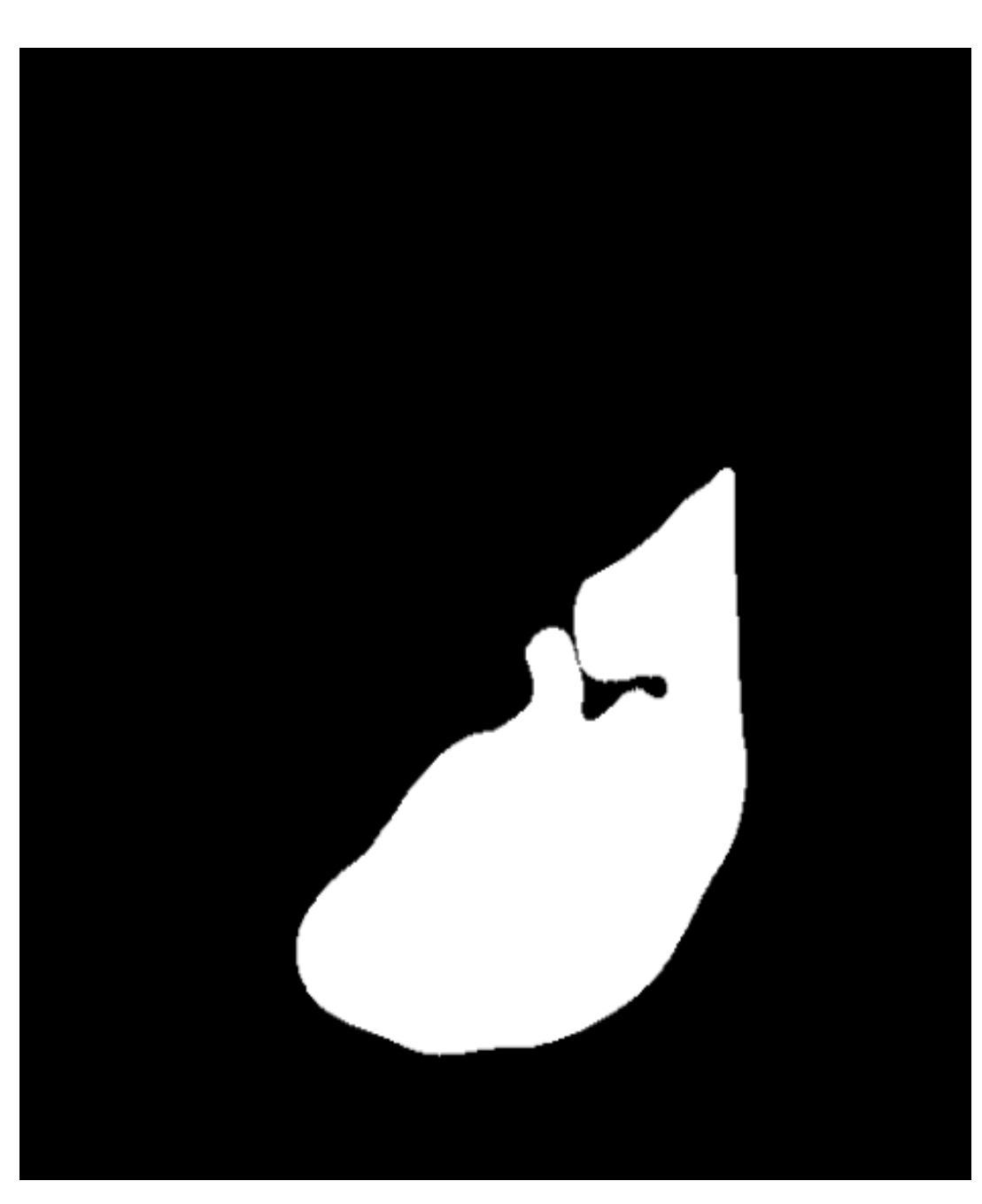

(a)

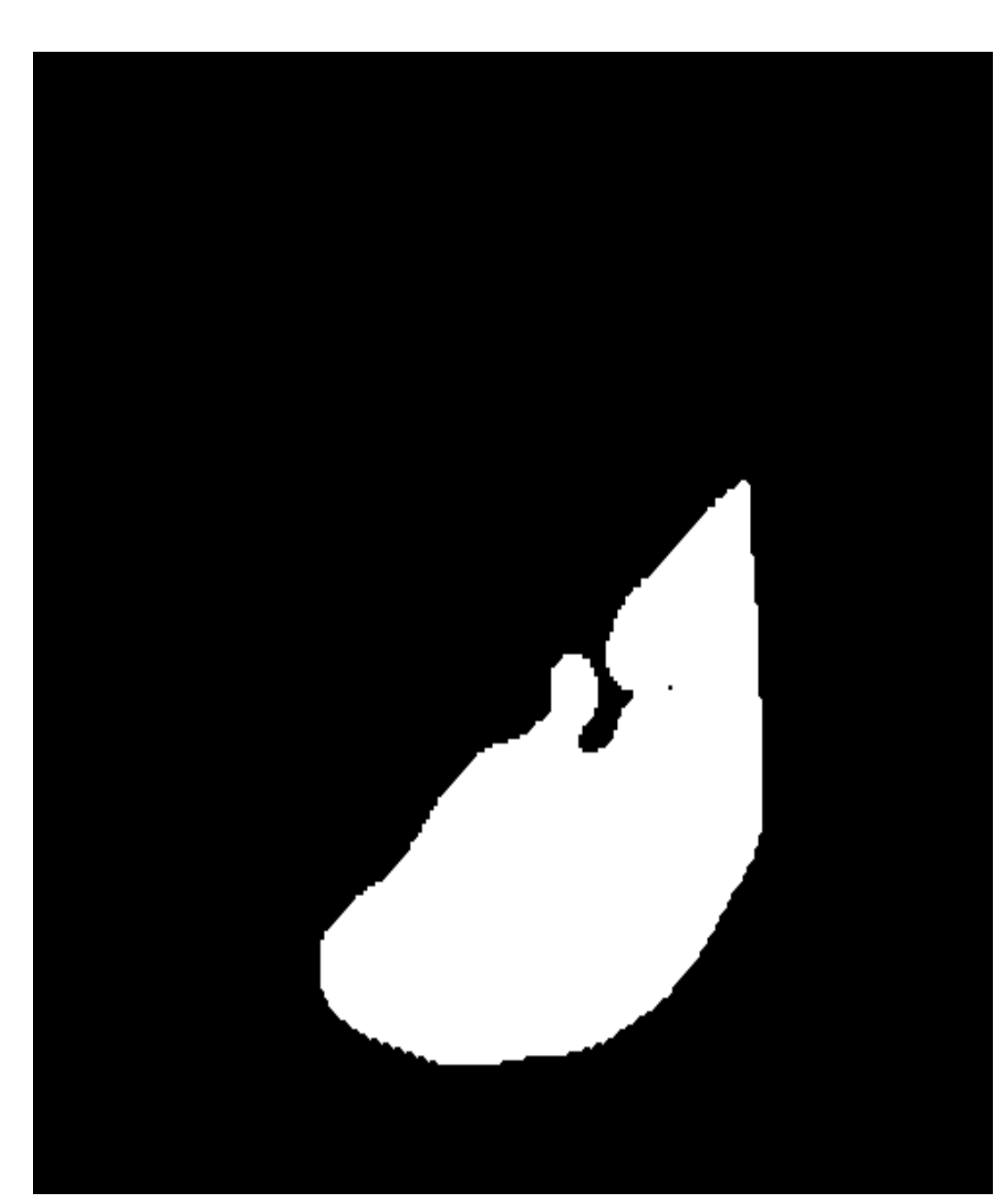

(b)
Figure 4: Slice 97 in record 294 MSDC-T8 (a) ground-truth (b) segmentation

Maximum epochs is equal to 75 , a batch size of 32 , and a learning rate $=16 \times 10^{-5}$ are used. Early stopping intervenes if the model's performance worsens on the validation set.

- A 3D model is created by interpolating the segmented slices via the marching cubes algorithm (Refer to Figure 5). The script can build multiple objects with different colors.

(a)

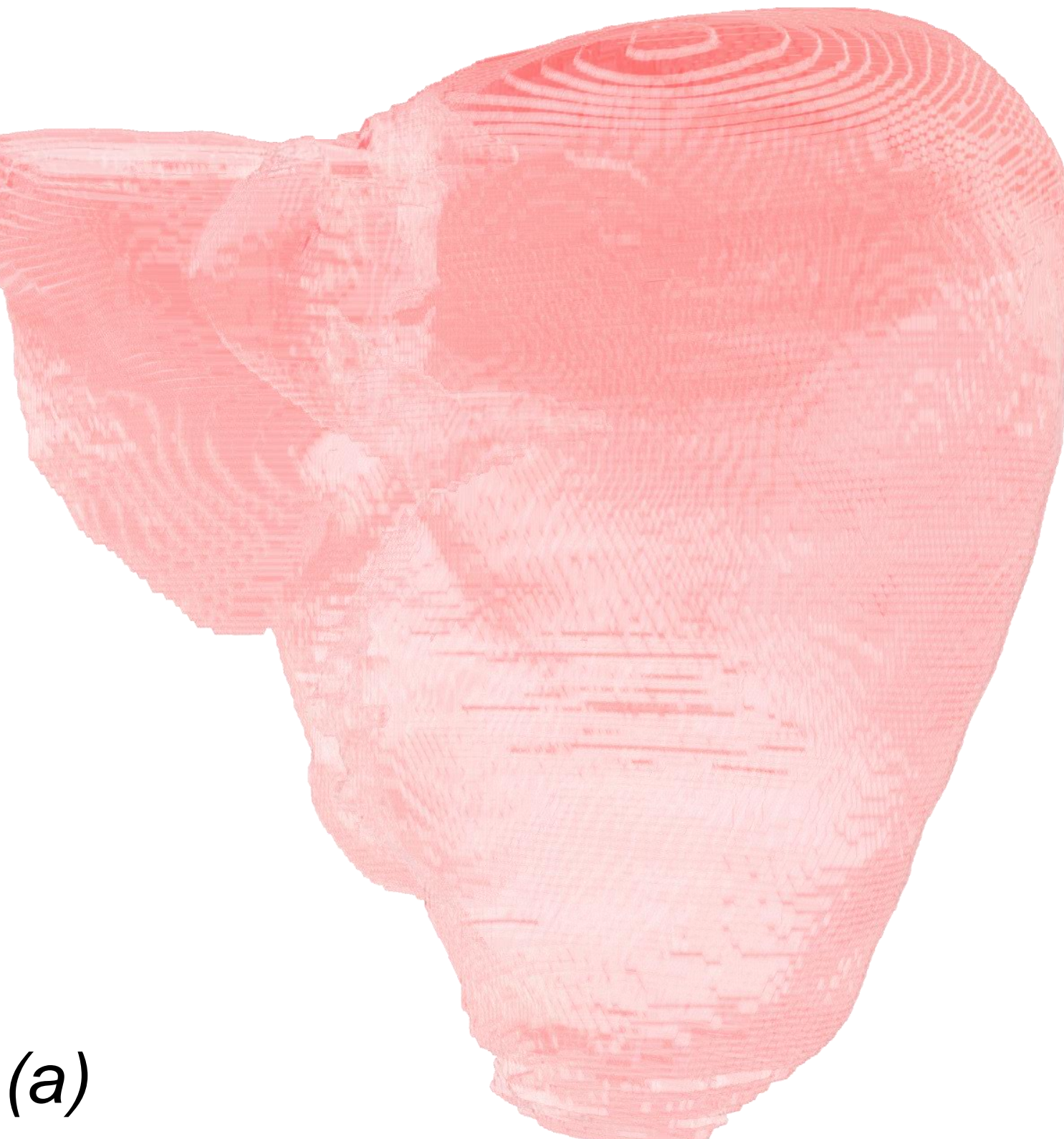

(b)

Figure 5: 3D rendered Liver volume in record 294

(a) ground-truth (b) segmentation

- Table 1 shows the average results of the best run from a 5 -folds cross-validation process over 23 selected unseen records.

Table 1: Classifications Results

$\begin{array}{llllllll}\text { Dice loU } & \mathrm{Pr} & \mathrm{Re} & \mathrm{Sp} & \text { ASD } & \text { RMSD } & \text { HD } & \text { 95\% HD }\end{array}$ 98.12\% 96.33\% 98.54\% 97.73\% 99.93\% 0.624mm 2.15mm 27.16mm $4.10 \mathrm{~mm}$

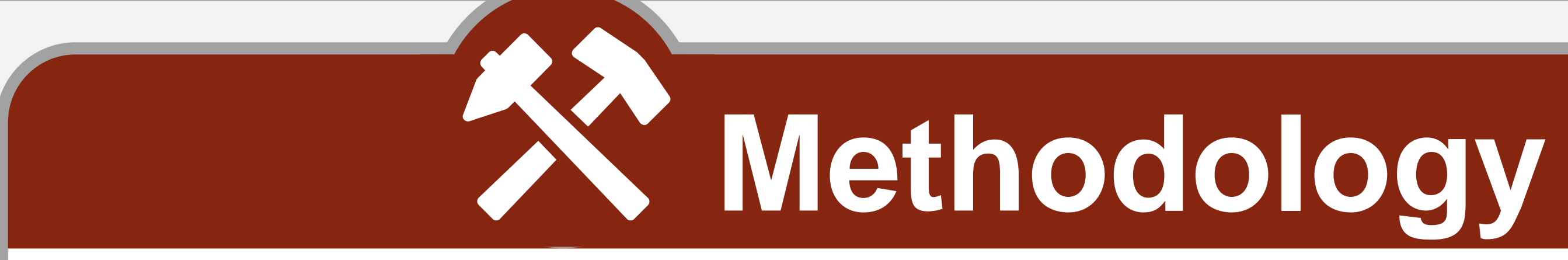

- The methodology contain multiple steps as seen in Figure 1.

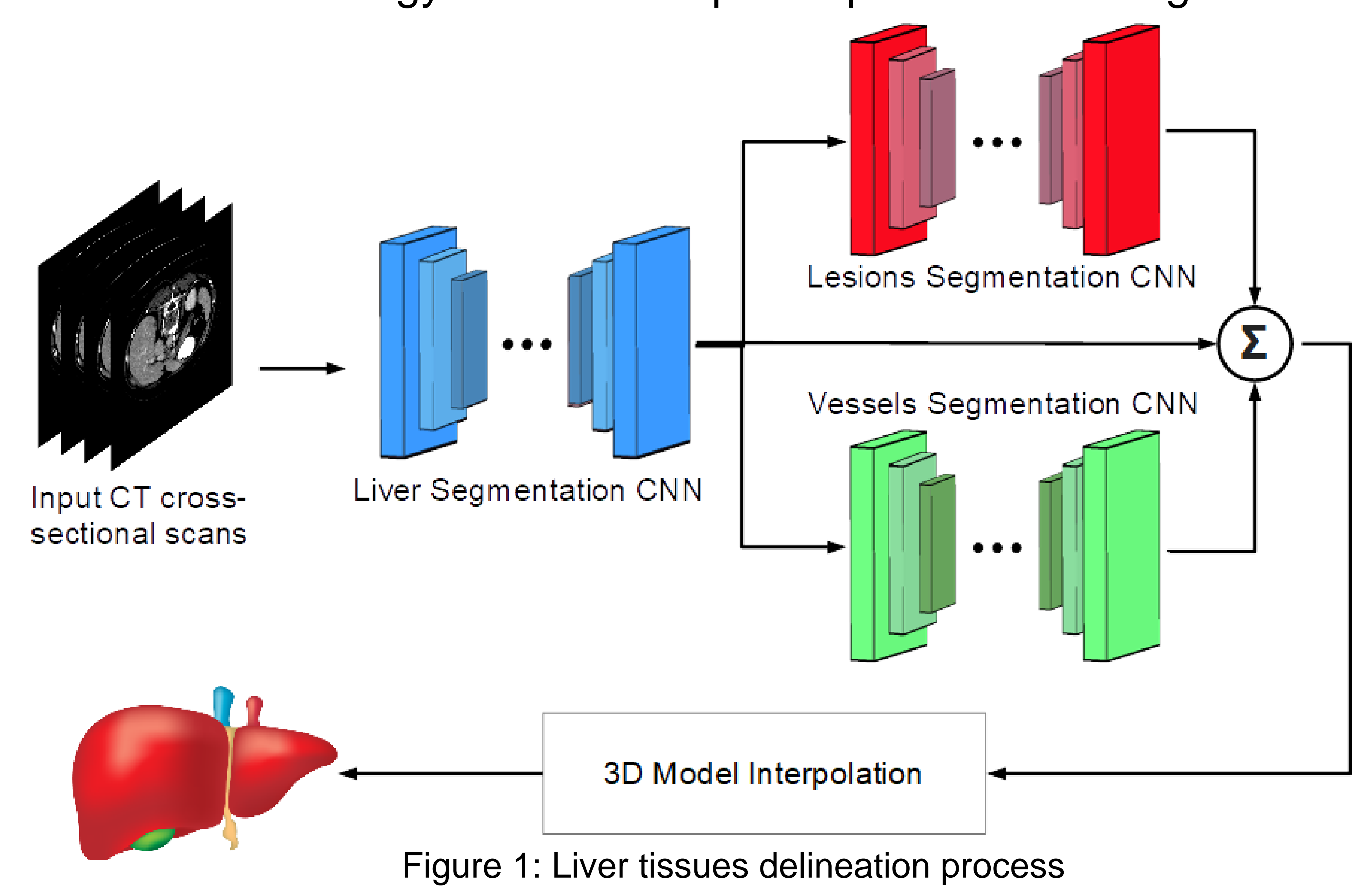

- An existing dataset is utilized to test the algorithm. It is from the Medical Segmentation Decathlon Challenge (Task 8: Hepatic Vessel), abbreviated as MSDC-T8.

- The CT records are preprocessed, as shown by Figure 2, before the first ConvNet used for liver segmentation.

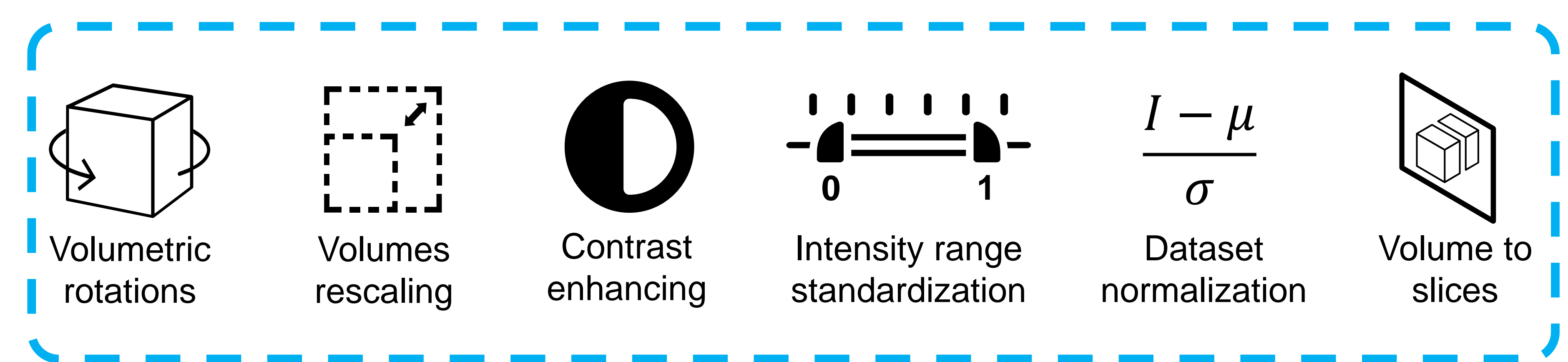

Figure 2: CT records pre-processing

- The slices are gathered into a $2.5 \mathrm{D}$ fashion by grouping odd adjacent slices, then outputting the delineation for the middle one (Refer to Figure 3).

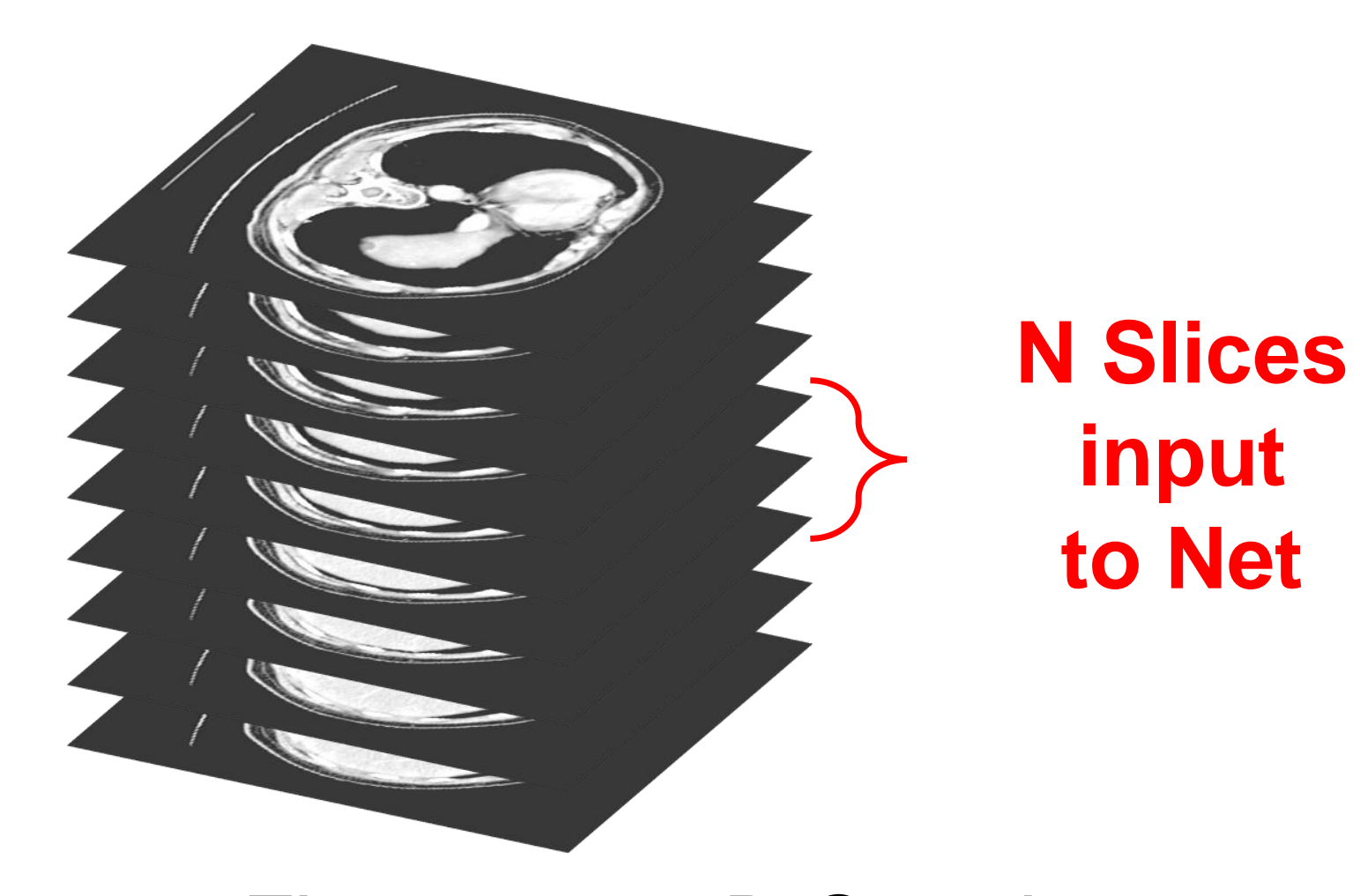

Figure 3: $2.5 D$ Creation

- Finally, after delineating the liver in a CT record, a 3D object is rendered from the segmented slices to create a liver for surgeons to examine.

\section{monclusion \& Future Work}

\section{Conclusions:}

- Created a U-Net ConvNet model that delineates liver from a contrast-enhanced CT scan.

- Achieved state-of-the-art results with respect to the original paper that published the liver masks for the MSDC-T8 dataset.

- Added 3D modeling aspect to the delineated liver.

\section{Future Work:}

- Add the tumor and vessels delineation models to segment these highly relevant tissues.

- Create a program that will take the record and segment all the tissues and render a 3D object for medical use via the click of a button. 